\title{
Pratiques
}

Linguistique, littérature, didactique

187-188 | 2020

Enseignement du texte littéraire dans l'espace

francophone: pratiques, formation, recherche

\section{Le sujet lecteur en formation d'enseignant'e's}

The reader subject in teacher training

Brigitte Louichon et Agnès Perrin-Doucey

\section{(2) OpenEdition}

Journals

Édition électronique

URL : https://journals.openedition.org/pratiques/8932

DOI : 10.4000/pratiques.8932

ISSN : 2425-2042

Éditeur

Centre de recherche sur les médiations (CREM)

Référence électronique

Brigitte Louichon et Agnès Perrin-Doucey, « Le sujet lecteur en formation d'enseignant.e.s », Pratiques [En ligne], 187-188 | 2020, mis en ligne le 18 décembre 2020, consulté le 21 juillet 2021. URL : http:// journals.openedition.org/pratiques/8932 ; DOI : https://doi.org/10.4000/pratiques.8932

Ce document a été généré automatiquement le 21 juillet 2021.

(c) Tous droits réservés 


\section{Le sujet lecteur en formation d'enseignant·e's}

The reader subject in teacher training

Brigitte Louichon et Agnès Perrin-Doucey

1 Depuis plus de 15 ans maintenant, le concept de sujet lecteur a été mis sur le devant de la scène en didactique de la littérature et a généré quantité de travaux de recherche. Dans un premier temps, nous exposerons la manière dont il est devenu outillant, discuté, disséminé, vulgarisé et préconisé. Autrement dit, nous observerons comment ce concept a migré du champ de la recherche à celui des recommandations. Nous proposerons ensuite de rendre compte d'une enquête réalisée auprès des formateur.trice's d'enseignante's, en posant que l'espace de la formation constitue un entredeux entre celui de la recherche et celui des pratiques d'enseignement/ apprentissage. Dans notre conclusion, nous nous appuierons sur quelques données relatives à un projet de recherche portant sur les pratiques effectives d'enseignement de la littérature afin de mettre en perspective nos résultats. En étudiant ainsi la circulation du concept et la manière dont il se transforme en objet de formation, nous observons qu'il occupe dorénavant une place dominante dans l'espace de la formation et nous nous interrogeons sur les effets potentiels d'une telle situation.

\section{Le concept de sujet lecteur}

2 Avant de retracer à grands traits l'histoire du sujet lecteur dans le champ de la didactique, posons clairement ce que nous entendons par concept, en adoptant la définition proposée par B. Daunay, Y. Reuter et B. Schneuwly (2011, p. 16) :

Nous entendons par concepts, ici, des syntagmes fonctionnant comme des moyens théoriquement construits, au sein de notre discipline, raisonnablement stables et opératoires, pour une appréhension du réel qu'elle vise à décrire, à expliquer ou à transformer.

Nous établirons d'abord qu'il existe une sorte de protohistoire de cette conceptualisation. Nous observerons ensuite l'élaboration du concept dans l'espace de 
la recherche, puis sa transformation en outil didactique avant de l'envisager dans une perspective d'outillage didactique.

\section{I.1. De l'expression au concept}

4 «L'archéologie, explique un commentateur de M. Foucault, s'applique à comprendre comment certains objets, au détriment de certains autres, ont pu apparaitre comme objets de connaissance" (Vuillemin, 2017). C'est une question que l'on peut légitimement se poser par rapport au sujet lecteur. Pour mémoire, et nous y reviendrons, on peut dater l'émergence du concept à un colloque tenu en 2004 et organisé par G. Langlade et A. Rouxel. Cependant, l'expression «sujet lecteur » ou encore celle de "lecture subjective" sont présentes avant que le concept ne soit au cœur des recherches et des discussions menées par les didacticiens de la littérature. Par exemple, dans un numéro de Pratiques de 1982, on trouve l'expression sous la plume de L. Sprenger-Charolles $(1982$, p. 3) : «On occulte complètement le rôle du sujet lecteur dans le processus de lecture : le pourquoi et le comment il lit». Toujours dans Pratiques, un article de M. Burgos (1992) ${ }^{1}$ parle de la lecture comme de l'activité d'un sujet, du "pôle texte» et du "pôle sujet ». Dans le champ de la didactique de la littérature naissante, les propos de R. Barthes (1984, p. 47) sur la lecture étaient assez présents : "Toute lecture procède d'un sujet, et elle n'est séparée de ce sujet que par des médiations rares et ténues, l'apprentissage des lettres, quelques protocoles rhétoriques, au-delà desquels, très vite c'est le sujet qui se retrouve dans sa structure propre, individuelle». R. Barthes et son "avènement du lecteur ", son appel à une théorie de la lecture sont clairement à l'origine de cette dynamique. Tout comme M. Picard (1986, p. 10) qui jouera un rôle majeur dans cette aventure théorique : "On escamote à l'aide du modèle de la communication à la fois le texte et le sujet. En particulier le sujet lecteur dont l'activité complexe excède de toutes parts, manifestement, le rôle de "décodeur" ".

5 En 2001, G. Langlade écrit un article important : «Et le sujet lecteur dans tout ça? ».

L'auteur y questionne l'enseignement de la littérature :

Les textes littéraires sont bien présents dans cet enseignement [...] mais cependant, ils ne semblent pas donnés à lire ni soumis à l'interprétation pour eux-mêmes, au $\mathrm{vu}$ de leurs richesses propres et des expériences de lecture individuelles et collectives qu'ils sont susceptibles de générer. Pour l'essentiel, les textes sont inféodés à des objets d'étude dont la maitrise par les élèves constitue de fait la finalité affichée de l'enseignement de la littérature [...]. Les œuvres [...] ne paraissent en quête d'aucun sujet lecteur, elles ne semblent prendre place dans aucune aventure de lecture, ne viser aucun retentissement intime ni aucune confrontation interprétative ... Les questions fondamentales - que disent, nous disent, me disent les œuvres littéraires ? À quoi servent-elles ? - semblent devenues superflues, voire incongrues, dans un tel enseignement de la littérature. (Langlade, 2001, p. 53-54).

6 Tout ce qui va constituer le fondement des travaux à venir y est: critique d'un enseignement post-lançonnien, formaliste et techniciste ; enjeux de l'enseignement de la littérature ; interprétation; dimension intime et collective de la lecture en contexte scolaire... Pour autant, dans cet article, l'expression « sujet lecteur » est employée, sans jamais être clairement définie et surtout sans jamais être référencée. Autrement dit, sans jamais être appréhendée comme un concept. 
7 En 2004, on passe de l'expression au concept, ou du moins au désir de conceptualisation lors du colloque intitulé : «Le sujet lecteur. Lecture subjective et enseignement de la littérature ${ }^{2} »$.

8 La question posée est celle du lecteur empirique, du lecteur réel, dont l'enseignement ne peut faire l'économie. Et dès l'introduction, G. Langlade et A. Rouxel (2004, p. 13) parlent de "questions scientifiques et didactiques»: ce double questionnement qu'est-ce qu'un lecteur réel et comment enseigner la littérature en prenant celui-ci en compte? - est une dimension absolument centrale dans l'histoire du concept. D'une part, les travaux vont se déplacer massivement du côté de la classe, mais d'autre part, demeure toujours la volonté de décrire le réel de l'activité lectorale. En 2007, G. Langlade et M.-J. Fourtanier signent un texte important «La question du sujet lecteur en didactique de la lecture littéraire " qui cherche à "élaborer un modèle opératoire de la lecture subjective ».

9 Si l'on poursuit cette généalogie du concept, le colloque de 2008, consacré au « texte du lecteur » (Mazauric, Fourtanier \& Langlade, 2011a ; 2011b), constitue un deuxième jalon de la construction collective de la notion dans le champ didactique. L'expression « texte du lecteur ", empruntée à J. Bellemin-Noël (2001, p. 21), désigne, dans le cadre du colloque, tout discours de réception subjectif ou intersubjectif, oral ou écrit, artistique ou critique, scolaire ou pas. En d'autres termes, le texte du lecteur constitue un domaine à explorer dans ses formes, ses fonctions, ses modalités et ses contextes d'émergence. Mais dans le même temps, et d'un point de vue méthodologique, il permet d'étiqueter l'ensemble des données permettant la description de l'activité réelle de lecture sous un vocable commun et unique. Par voie de conséquence, à partir de cette date, il constitue scientifiquement un marqueur fort d'appartenance à la communauté scientifique du sujet lecteur.

On peut aussi constater, dans les deux volumes d'actes du colloque, une dichotomie que marquent les titres : «Le texte du lecteur " pour le volume 1 et « Textes de lecteurs en formation " pour le volume 2. Leurs éditeurs indiquent que le premier s'est attaché à "répondre aux questions les plus générales [...] que soulève le concept » tandis que le second est consacré à la lecture scolaire (Mazauric, Fourtanier \& Langlade, 2011b, p.13). On retrouve la dichotomie des «questions scientifiques et questions didactiques». Il apparait donc qu'entre 2004 et 2008, le concept de sujet lecteur continue à s'élaborer scientifiquement, notamment grâce à l'articulation, à la fois conceptuelle et méthodologique, avec celui de texte du lecteur. Dans le même temps, il commence à outiller les recherches.

\section{2. Du concept à l'outil didactique}

11 La notion d'outil didactique est empruntée à B. Daunay (2007a, p. 168) : «Dire de la notion de lecture littéraire qu'elle est un outil didactique, c'est signaler son intérêt et sa productivité dans les recherches didactiques concernant l'enseignement de la littérature ». On peut dire exactement la même chose du sujet lecteur. De nombreuses thèses, soutenues depuis une dizaine d'années - travaux que l'on peut qualifier «de seconde génération »- articulent une double finalité : expérimenter des dispositifs didactiques susceptibles de prendre en compte la dimension subjective du lecteur scolaire et décrire l'activité de ce lecteur réel, autrement dit se poser des questions "scientifiques et didactiques ». Ces travaux dont le cadrage théorique donne une place 
déterminante au sujet lecteur sont menés à des niveaux différents (du cours préparatoire au lycée général et professionnel); dans des pays francophones différents (France, Québec, Suisse, Belgique); et travaillent des notions diverses (la spectature, l'identité de lecteur, la diversité du sujet lecteur, l'évènement de lecture, l'appropriation, l'empathie fictionnelle, l'appréciation, la participation) ${ }^{3}$. À partir de ces entrées sont observées des pratiques ordinaires ou mis en place des dispositifs expérimentaux. Les résultats sont triples quant à leur nature :

- les notions sont configurées dans une perspective didactique ;

- la manière dont lisent les élèves, les enjeux qu'ils assignent à la lecture des œuvres, la manière dont les effets se déploient dans un temps long, les difficultés que les élèves comme les enseignant·e's rencontrent sont décrites et interprétées ;

- des dispositifs sont expérimentés ou ré-expérimentés, analysés, évalués, discutés, comparés. peut constituer le seul horizon de la lecture scolaire, d'autre part parce qu'elle pourrait conduire à minorer, voire à dénaturer la place du texte et de la culture dans la relation lectorale, enfin parce qu'elle serait la porte ouverte au "subjectivisme flou» « débridé » (Daunay, 2007b) et au relativisme ${ }^{5}$.

17 Ces discussions demeurent aujourd'hui (Dufays, 2016), signe de la vitalité d'un concept qui continue à se déplacer vers le terrain.

\section{3. De l'outil didactique à l'outillage didactique}

18 En effet, l'usage du concept dans le champ de la recherche s'est disséminé en direction

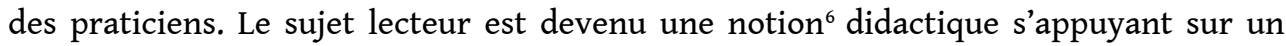


substrat théorique, notion discutée, institutionnalisée, vulgarisée et susceptible d'applications pédagogiques.

Pour ce qui est de la vulgarisation, dans l'ouvrage dirigé par S. Ahr (2013), Vers un enseignement de la lecture littéraire au lycée, le cadrage théorique fait une place essentielle au sujet lecteur et l'expression est utilisée très fréquemment dans l'ouvrage, tout comme celle de «lecture subjective».

Pour ce qui est de l'institutionnalisation en France, un texte d'A. Vibert, inspectrice générale de lettres, datant de 2011, est disponible sur Éduscol comme « ressource pour le collège et le lycée ». Il s'intitule «Faire place au sujet lecteur en classe : quelles voies pour renouveler les approches de la lecture analytique au collège et au lycée ?». On le voit, le titre institutionnalise la notion et lui confère une visée transformative: «L'objectif, écrit l'autrice, est bien désormais de sortir des expériences ponctuelles et de passer à une phase d'application plus générale de ces propositions pour voir dans quelle mesure elles répondent à nos préoccupations et sont conciliables avec les objectifs des programmes ». Les programmes français pour les cycles 2 , 3 et $4^{7}$ (Ministère de l'Éducation nationale, de la Jeunesse et des Sports, 2015) développent cette approche :

Les activités de lecture mêlent de manière indissociable compréhension et interprétation. Elles supposent à la fois une appropriation subjective des œuvres et des textes lus, une verbalisation de ses expériences de lecteur et un partage collectif des lectures pour faire la part des interprétations que les textes autorisent et de celles qui sont propres au lecteur (ibid., p. 108).

21 Outre les échos que suscite la terminologie nouvelle utilisée, on remarquera que, pour le cycle 3 et le cycle 4 , les finalités de l'enseignement de la littérature conjoignent des «enjeux littéraires et de formation personnelle». C'est un saut considérable dans l'explicitation des enjeux de la littérature enseignée. En effet, le sujet est à la fois celui qui actualise singulièrement le texte, qui en construit la signification, mais aussi celui qui se construit par sa lecture. D'une certaine manière, on peut considérer que les postulats des chercheurs travaillant sur le sujet lecteur innervent ces programmes: place du lecteur, singularité des interprétations et des utilisations, tension entre droits du texte et droits du lecteur, communautés interprétatives, enjeux anthropologiques de la lecture littéraire...

22 Pour être tout à fait honnête, force est de constater que les programmes de 2018 pour les cycles 3 et 4, s'ils conservent les enjeux de formation littéraire et de formation personnelle, ont fait disparaitre tout ce qui pouvait ressortir au sujet lecteur dans la terminologie adoptée (Ministère de l'Éducation nationale, de la Jeunesse et des Sports, 2018). De même, les programmes pour le lycée professionnel sont quasiment désertés par toute l'ambition qui portait les précédents (Belhadjin, 2009). Curieusement, la lecture des programmes du lycée général 2019 réservent quelques surprises. On y parle en effet de compétences "de lecture et d'interprétation", et même d'appropriation, cette compétence s'exerçant et se construisant grâce à ce que les programmes nomment précisément "écrits d'appropriation " (Ministère de l'Éducation nationale, de la Jeunesse et des Sports, 2019b). Or cette notion a été développée par B. ShawlkyMilcent (2020) à partir du concept de sujet lecteur.

23 À travers ce bref et nécessairement schématique historique du sujet lecteur ${ }^{8}$, nous avons montré comment le sujet lecteur est devenu un concept productif et discuté, outillant la recherche en didactique, vulgarisé, institutionnalisé jusqu'à innerver les 
programmes scolaires français. La formation des enseignante-s se nourrit, pour partie, des résultats de la recherche et vise à doter les futurs professionnels des savoirs et compétences nécessaires à l'exercice de leur métier. On peut dès lors se demander quelle place le sujet lecteur occupe dans cet espace intermédiaire entre recherche et pratique professorale.

\section{Analyse des déclarations des formateur-trice-s}

\section{II.1 Questionnaire et profils des répondant·e·s}

Pour répondre à cette question, nous avons créé un questionnaire en ligne ${ }^{9}$ (voir annexe 3) composé d'une majorité de questions fermées, associées le plus souvent à un espace de commentaire ouvert permettant de justifier ou préciser les réponses fournies. Le questionnaire a été diffusé par voie électronique aux enseignant·e's des différents organismes francophones de formation. Celui-ci est composé de trois sections : 1) profil ; 2) formation et modalités de formation ; 3) commentaire personnel. L'étude se construit donc sur la base d'une déclaration volontaire des formateur.trice's et ne permet en aucun cas d'interpréter les pratiques. En revanche, elle est à même de renseigner un ensemble de représentations. Notre corpus d'analyse est constitué de 69 réponses de formateur.trice-s intervenant en France métropolitaine à minima auprès de futurees enseignantees du second degré, et pour un peu plus de la moitié des réponses recueillies, dans le premier et le second degrés. L'échantillonnage étant construit de façon écologique, il n'est pas nécessairement représentatif de l'ensemble de la profession. Cependant, l'ensemble des catégories statutaires intervenant en formation est représenté ${ }^{10}$ dans des proportions significatives, il en va de même pour le décompte des années d'expérience. La formation initiale suivie par les participant·e-s est majoritairement ancrée dans le champ littéraire (611) ou linguistique (6). Les sciences de l'éducation sont aussi représentées (12) mais le plus souvent au sein de doubles cursus qui allient surtout le champ des études littéraires (9). Les autres réponses s'avèrent assez éloignées des disciplines majoritaires dans notre corpus (Corse, Histoire, Philosophie, sciences de gestion, technologie-sciences de l'ingénieur). Une partie des répondante-s (37) exercent une activité de recherche, située pour 21 répondant·es dans le champ littéraire et didactique ou pédagogique ${ }^{12}$.

\section{II.2 Présence ou non de la notion de sujet lecteur dans la formation}

La section intitulée "formation et modalités de formation " propose une première question obligatoire : La notion de sujet lecteur est-elle présente dans vos formations? Pour répondre, les participants ont le choix entre trois items: oui explicitement; oui implicitement; non.

Le sondage met clairement en évidence une présence de la notion de sujet lecteur dans la formation. En effet, 60 personnes affirment aborder la question explicitement (50), ou implicitement (10). Seules neuf indiquent l'absence de la notion dans leurs formations.

L'étude des profils de cette dernière catégorie laisse supposer que la formation initiale a une incidence sur ce choix. En effet, environ la moitié des répondante's a suivi une formation très éloignée du domaine lettres et langue : comme les sciences de gestion 
(1), la technologie-sciences de l'ingénieur (1), le Corse (1), ou la philosophie (1). Les autres réponses se répartissent entre les sciences du langage (1), les sciences de l'éducation (1), les langues anciennes (1) et la littérature (2). On observe aussi qu'aucune personne ayant répondu non n'exerce une activité de recherche en didactique de la littérature, ni même en littérature. Qu'en est-il des personnes ayant répondu oui à la question? Note-t-on des différences de profils entre les choix explicite et implicite?

La corrélation des réponses aux dimensions statutaire ou expérientielle des répondante's n'apporte aucune information pertinente pour expliquer ce choix. En revanche, les indications concernant leur formation initiale, comme les domaines dans lesquelles s'effectuent des activités de recherche mettent en évidence quelques petites divergences. La majorité des répondant-e's a suivi un cursus de formation initiale en littérature : $96 \%$ du groupe explicite et $90 \%$ du groupe implicite. Une étude plus précise montre que $20 \%$ des personnes affirmant développer une approche explicite a suivi un double cursus littérature et sciences de l'éducation, alors qu'il n'y en a point dans le groupe implicite. Nous avons aussi corrélé les réponses à l'activité de recherche exercée. $54 \%$ du groupe explicite et $50 \%$ du groupe implicite exercent une activité de recherche. Les champs de la didactique et de la pédagogie représentent $67 \%$ des réponses issues du groupe explicite, les autres travaillant en littérature. $60 \%$ des réponses issues du groupe implicite déclare un ancrage en littérature générale, $20 \%$ en didactique des langues anciennes, et $10 \%$ dans un autre champ. Ces derniers chiffres doivent être relativisés dans la mesure où ils s'exercent sur de petits nombres de répondante's. Cependant, ces quelques éléments d'analyse nous permettent déjà de dresser un premier constat. La notion de sujet lecteur semble légèrement plus évidente chez les formateur.trice's croisant les disciplines littéraires et pédagogiques et/ou conduisant une activité de recherche en didactique et sciences de l'éducation.

29 L'analyse des choix bibliographiques et des modalités de formation, puis celle des activités pratiquées devraient nous permettre d'enrichir cette description.

\section{II.3 Analyse de la bibliographie}

Une bibliographie de 24 références (voir annexe 2) a été proposée par deux fois dans le questionnaire aux 60 personnes qui affirment la présence, explicite ou implicite, de la notion dans leurs cours. Elles sont invitées à cocher les références utilisées pour préparer leurs cours ${ }^{13}$ d'une part, et les références conseillées aux étudiant·e: $\mathrm{s}^{14} \mathrm{~d}$ 'autre part. Pour Préparation on relève 425 citations, chaque proposition est cochée au minimum 1 fois et au maximum 42 fois, soit une moyenne de 7 citations par questionnaire. Pour Étudiants, on relève 279 citations, allant de l'absence d'une référence (Dumayet) à 46 citations, soit une moyenne de 5 citations par questionnaire. Pour le groupe explicite, les références utilisées dans la préparation sont en moyenne de 8 citations au lieu de 7. Nous avons ensuite calculé le nombre de citations exprimé en pourcentages, pour les cinq titres les plus souvent représentés dans quatre contextes différents: Préparation et Étudiants pour l'ensemble du corpus traité (Préparation ensemble - Étudiants ensemble) et la même chose pour le groupe spécifique explicite (Préparation explicite - Étudiants explicite). Le graphique ci-dessous présente les différents résultats, que nous analysons ci-après. 
Graphique 1 : Références bibliographiques utilisées et recommandées

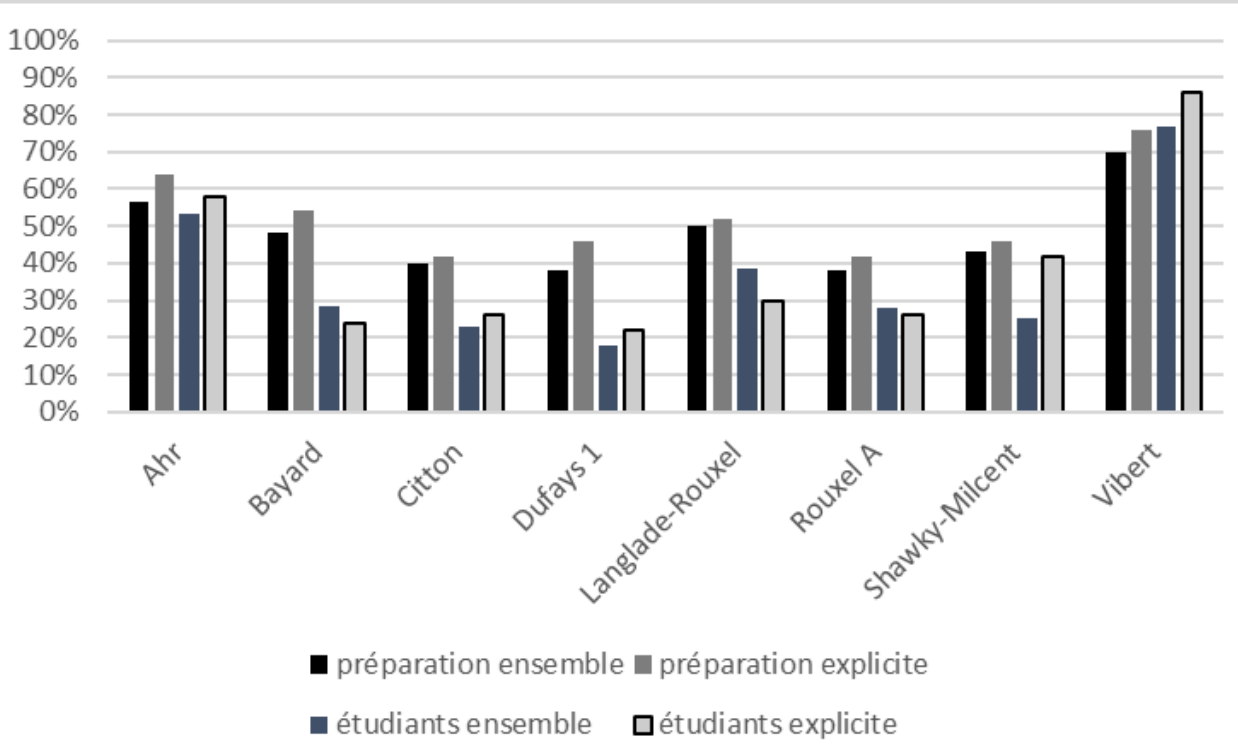

Quand on cumule les références choisies par les groupes explicite et implicite, on observe qu'elles sont identiques bien qu'ordonnées différemment pour Préparation et Étudiants. En revanche, le référencement du groupe explicite varie légèrement pour Étudiants. L'ouvrage de P. Bayard n'est pas cité, contrairement à celui d'Y. Citton et à l'article de J.-L. Dufays qui le sont. Dans tous les cas, les travaux plébiscités correspondent à des références institutionnelles (Vibert), aux recherches en didactique de la littérature (Ahr, Dufays, Langlade \& Rouxel, Rouxel, Shawky-Milcent), ou à des travaux théoriques ou critiques sur lesquels s'appuient les didacticiens (Bayard, Citton). Sans surprise, les outils institutionnels ou proposant des dispositifs prédominent dans les conseils donnés aux étudiante-s (Vibert, Ahr notamment). Ce classement des ouvrages les plus cités pourrait laisser penser que la notion est stabilisée sur le plan des références. Cependant, un espace permettant de compléter les propositions est ouvert à l'issue de chaque bibliographie. L'analyse des citations insérées par $30 \%$ des participant-ess (du groupe explicite) modifie un peu cette première interprétation, du fait de l'éclectisme des conceptions exprimées. En effet, les ajouts sont très importants pour Préparation (80 citations supplémentaires); un peu moins présents pour la partie conseils avec environ 25 propositions ajoutées. Parmi les ajouts proposés, nous relevons les travaux: de didacticiens autour de la lecture littéraire et la lecture subjective (Ahr \& Joole, ShawkyMilcent, Langlade, Rannou, etc.) ; de théoriciens de la lecture (Picard, Jouve, Bayard, etc..) dont les travaux ont nourri la construction du concept ; sur la dimension multimodale de la lecture (Boutin-Lacelle-Lebrun; Lacelle); en sémiotique et narratologie (Barthes, Eco, Baroni, etc.); en sciences humaines et sociales (Foucault, Lahire, Goody, etc.) ; sur la compréhension de texte (Goigoux \& Cèbe, Giasson) et son articulation à l'écriture.

L'analyse des réponses des 21 personnes du groupe explicite exerçant une activité de recherche ne permet pas d'établir un constat supplémentaire. Nous remarquons donc que les ajouts engagent une ouverture assez générale aux théories de la lecture et de la compréhension en lecture. L'analyse du questionnement à propos des bibliographies n'est pas en mesure de confirmer ou d'infirmer notre première observation. En effet, au regard de l'ensemble des citations cochées et des ajouts réalisés, il parait difficile de 
repérer ce qui relève spécifiquement de la notion de sujet lecteur pour les formateur.trice's ayant répondu à l'enquête.

\section{II.4 Analyse des modalités de formation proposées}

Pour mettre en évidence les modalités de formation, nous avons posé la question "Quelle(s) modalité(s) de formation à la notion de sujet lecteur utilisez-vous ?» et proposé un choix multiple de sept réponses présentées ci-dessous et restituées par le logiciel avec les mentions oui si l'item est coché, non s'il n'est pas coché. Les propositions s'organisent de la façon suivante :

- item 1 = cours théorique(s) ;

- item 2 = mise(s) en situation de l'étudiant comme « sujet lecteur ";

- item 3 = analyse(s) de situation(s) de classe(s) (vidéo ou audio) ;

- item 4 = analyse de séquence(s), séance(s) ou activité(s) ;

- item 5 = préparation(s) de séquence(s), séance(s) ou activité(s) ;

- item 6 = analyse(s) d'écrit(s) d'élève(s) ;

- item $7=$ autre $^{15}$.

Nous avons relevé 242 réponses pour les 60 répondant·e·s, soit en moyenne, 4 items sur les 6 choisis par personne. Le nombre de sélections d'un item varie d'un minimum de $32 / 60$ à un maximum de 48/60 sur l'ensemble du panel. Comme pour la bibliographie, nous avons souhaité comparer les résultats pour les groupes explicite et implicite. Il apparait que le nombre moyen de sélections par item est beaucoup plus élevé pour le groupe explicite $(4,5)$ que pour le groupe implicite $(2,8)$. Il semble donc intéressant de comparer la répartition de ces choix par items pour chaque groupe. Le graphique 3 cidessous indique le pourcentage de personnes ayant émis la réponse oui pour un item donné.

\section{Graphique 2 : Modalités de formation}

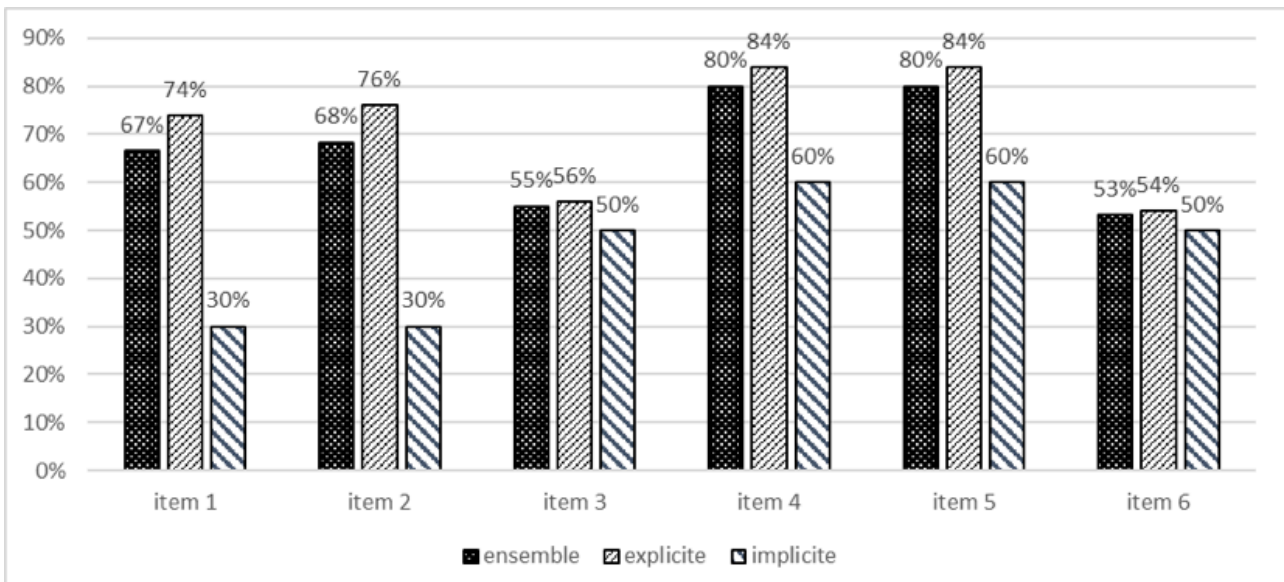

Le classement des items choisis permet de repérer des invariants et des variations entre chacune des deux approches. L'analyse (item 4) et la préparation (item 5) de séquences, de séances, ou d'activités sont les deux propositions qui ont recueilli le plus de oui et semblent donc être les plus prisées en formation, quelle que soit l'approche de la notion choisie. Les autres modalités liées à l'analyse de situations (audio-vidéo = item 3) ou d'écrits d'élèves (=item 6 ) sont choisies de façon équivalente pour chacun des deux 
groupes, même si elles restent les moins prisées pour le groupe explicite. L'item 6 (écrits d'élèves) reste le moins choisi. Les variations les plus importantes se situent pour les items 1 (cours théoriques) et 2 (mises en situation des étudiants). Le pourcentage pour le groupe explicite est nettement plus élevé que pour le groupe implicite. Cette variation semble intéressante car elle pourrait justifier le choix de l'expression « explicite " par une plus grande prise en compte scientifique et pratique de la notion. En effet, il s'agit là de mettre les formée's en situation de sujets lecteurs et d'aborder les concepts théoriques qui définissent la notion. Cependant, il y a quelques limites à cette interprétation. Certes le pourcentage est légèrement plus élevé pour le groupe explicite, néanmoins la variation reste peu signifiante statistiquement. En effet, elle peut s'expliquer, d'une part parce que le groupe implicite a sélectionné en moyenne nettement moins d'items : la dispersion est donc plus visible; d'autre part parce qu'il est constitué d'une population moins nombreuse, ce qui influe sur la représentativité des pourcentages. Malgré ces réserves, nous pouvons affirmer que ces légères variations restent cohérentes avec notre premier constat: le groupe explicite semble plus à l'aise pour prendre en compte la notion en formation que le groupe implicite.

Nous avions établi notre premier constat en supposant que le cursus initial et l'ancrage scientifique de leurs activités de recherche avaient une influence sur les réponses. Nous avons donc poursuivi cette investigation en découpant le groupe explicite en deux sous-groupes en fonction de la description des activités de recherche: exp-hors recherche et exp-recherche ${ }^{16}$. Nous constatons tout d'abord que la moyenne du nombre d'items choisi par personne, augmente pour exp-recherche $(4,9)$ et diminue pour exp-hors recherche $(3,9)$. Le graphique 3 ci-dessous retrace les données de la quantification en pourcentages par items et par répondant·e's.

Graphique 3 : Modalités de formation et expérience de la recherche

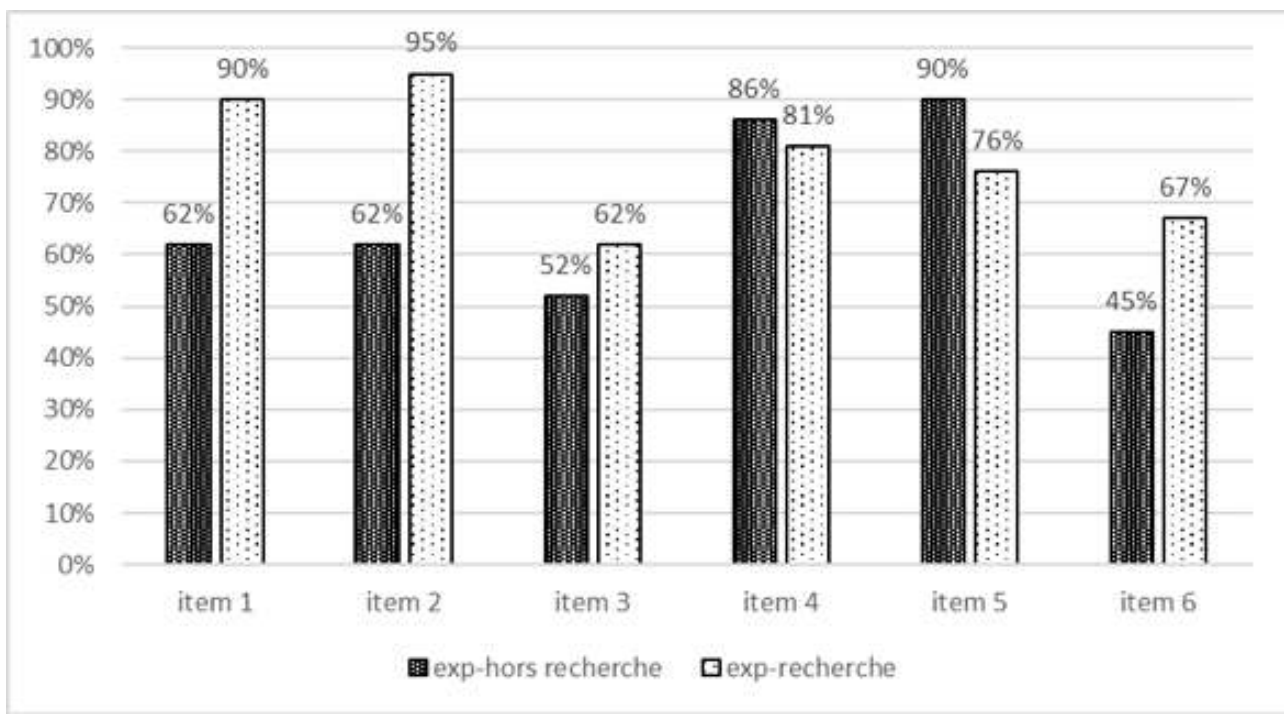

Les pourcentages pour exp-recherche sont globalement en hausse puisque le nombre de propositions est plus élevé pour ce groupe. Ce qui est intéressant, c'est la variation d'ordre qui apparait. L'activité visant à mettre l'étudiantee en situation de sujet lecteur est la plus choisie, contrairement aux groupe exp-hors recherche et implicite, puis viennent les cours théoriques dans un volume équivalent aux productions de séquences, séances ou activités. On peut donc considérer que les modalités mobilisées 
par ces formateurtrice's visent plus spécifiquement la construction de connaissances scientifiques et de dispositifs didactiques qui mobilisent une expérience de l'étudiante.e. Les données extraites des réponses du sous-groupe exp-hors recherche diffèrent de façon assez importante pour se rapprocher des résultats constatés avec le groupe implicite.

À ce stade de notre recherche, nous pouvons donc confirmer la corrélation établie dans notre premier constat entre l'activité de recherche conduite par les répondanteess et la manière dont est abordée la notion en formation. La réponse oui explicite apparait comme moins signifiante quand on se concentre sur les seules données du sous-groupe exp-hors recherche. Cependant, il faut là-encore nuancer ces propos, car si les répondante-s du groupe exp-recherche semblent choisir majoritairement des modalités directement liées à la notion analysée, ils ou elles n'excluent pas les autres. La dispersion des propositions réalisées reste donc perceptible quels que soient les profils des répondant·es, elle s'accroit même sensiblement pour le sous-groupe exp-recherche. Leurs propositions ne se focalisent pas sur une ou deux modalités spécifiques, mais englobent plusieurs réponses dont les visées sont assez différentes.

L'analyse des activités proposées par les participante's permet-elle d'aller plus loin dans ces observations?

\section{II.5 Analyse des activités déclarées par les participant·e·s} espace de commentaire possiblement long: "Quelle(s) activité(s) d'enseignementapprentissage en lien avec le sujet lecteur proposez-vous ?». Nous avons reçu 37 réponses pour 60 personnes ayant accès à la question. 2 réponses proviennent du groupe implicite, 13 réponses du sous-groupe exp-recherche, 12 réponses du sous-groupe exp-hors recherche. Pour cette étude, compte tenu du constat réalisé précédemment, nous avons comparé les réponses du sous-groupe exp-recherche (= corpus 1$)$ et celles du sous-groupe exp-hors recherche auquel nous adjoignons, compte tenu du peu de variations repérées entre les deux, les deux réponses du groupe implicite (= corpus 2). La formulation a généré une ambigüité qui n'a pas permis d'obtenir une homogénéité des réponses. En effet, une partie d'entre elles concerne plus la pratique en formation et les activités proposées aux étudiant·e's en formation que celles destinées aux élèves. Cependant, la dimension ouverte du questionnement permet d'éclairer un peu la manière dont la notion de sujet lecteur est perçue.

41

L'ensemble des données recueillies comprend environ 1200 mots, 760 pour le corpus 1 et 440 pour le corpus 2 . Nous avons réalisé une analyse sémantique via le logiciel de lexicométrie Tropes ${ }^{17}$ qui nous a permis de mettre en évidence les récurrences lexicales au sein des corpus, puis d'établir des corrélations sémantiques pour tenter de proposer une typologie des réponses. Cette étude lexicographique contribue à démontrer l'éclectisme des propositions. L'analyse réalisée à partir d'un relevé des occurrences lexicales et des champs sémantiques liés aux fréquences d'utilisation, met en évidence la constitution d'un univers de référence reliant des éléments assez disparates. Il s'organise autour des mots "lecture" et "lecteur» qui sont les plus utilisés : 39 occurrences dans le corpus $1^{18}$ et 21 occurrences pour le corpus $2^{19}$. Le mot « sujet » leur est 11 fois associé dans le corpus 1, mais n'apparait jamais dans le relevé du corpus 2 . Enfin, le vocable « écrit » déterminant le champ sémantique de l'écrit et de l'écriture apparait en seconde position, (corpus $1=22$; corpus $2=15$ ).

Pratiques, 187-188 | 2020 

ainsi d'éclairer les liens qui peuvent se tisser entre les expressions utilisées. Nous avons réussi à déterminer quatre réseaux sémantiques au sein de cet univers de référence. Ils associent soit des outils ou modalités de travail mobilisés pour réaliser l'activité de lecture, par le lecteur-trice et l'enseignante's; soit des informations autour des pratiques mises en œuvre, soit des termes liés au champ professionnel ("élèves", « enseignants ", etc.) que nous ne prendrons pas en compte ici. Certains termes, peu nombreux, renvoient aux outils pensés pour mettre en évidence la présence d'une subjectivité de lecture, ou pour provoquer une expression personnelle du sujet (« carnet », « journal », « cercle », «biographie »). D'autres ont une connotation plus large liée à l'idée de "type d'écrit » développée dans le contexte d'apprentissage du français («lettre», « courrier», «texte»). D’autres encore concernent des médias utilisés en production ou réception («vidéo », « image », « manuscrit »). Parmi les types de tâches évoqués, on repère essentiellement des occurrences désignant la mise en œuvre de la pratique scolaire de la lecture des textes littéraires ("questionnaire», « débat », « commentaire », « analyse », « hypothèse », « interprétation »). Enfin l'étude montre que l'activité spécifique de l'élève peut être sollicitée, notamment pour lui permettre d'accéder à la compréhension. Ces activités semblent viser les dimensions cognitives de la compréhension et l'interprétation (" analyse »; "interprétation »; " compréhension»; "restitution »). L'interprétation est associée à des outils ou dispositifs (« débat», "hypothèse ») et à la notion de réception, qui reste peu développée.

L'analyse des activités confirment certains constats réalisés précédemment. Si la notion de sujet lecteur est prise en compte par les répondantees, elle semble incluse dans un ensemble plus vaste qui englobe la formation à l'enseignement du lire-écrire avec, par, ou pour le littéraire. On observe par exemple que si le terme "réception " est utilisé pour évoquer une fonction du carnet de lecteur, il est aussi associé à " question » et «questionnaire", mais jamais à "débat ». De même, l'idée de créativité transparait dans la dénomination de supports pour les activités d'écriture qui associent des formes de la réception («bande annonce», "titre» par exemple), mais elle reste peu développée. Les termes "subjectivité ", " appropriation » qui peuvent être considérés comme des mots-clés de la notion n'apparaissent ni explicitement, ni par le jeu des corrélations sémantiques dans aucun des deux corpus.

L'analyse des propositions révèle donc les confusions et peut-être des difficultés quand on demande de cibler plus spécifiquement les activités précises pour prendre en compte le sujet lecteur en formation. Sa présence semble être associée à l'enseignement de la lecture de la littérature, dans ses dimensions cognitive, métacognitive, réflexive, culturelle, etc. La notion est présente, légèrement plus affirmée pour les répondant·es exerçant une activité de recherche en didactique et pédagogie, mais elle apparait comme diluée dans le reste des notions travaillées en formation. 


\section{Conclusions}

\section{1 Le sujet lecteur : un concept glouton}

45 De cette enquête, nous retirons quelques lignes de force. La première concerne la présence affirmée de manière massive de la notion dans les contenus de formation ( $87 \%$ des personnes interrogées), quels que soient l'itinéraire des formateur-trice's et leur propre expérience de la recherche, même si quelques différences ont pu être observées de ce point de vue. La deuxième renvoie au fait que la notion se décline de manières très différentes dans les pratiques déclarées de formation comme dans les pratiques recommandées d'enseignement/apprentissage. La troisième porte sur les appuis bibliographiques convoqués pour former à la notion. Les trois références les plus proposées aux étudiant-e's sont le texte d'A. Vibert et le livre de S. Ahr - dont on a montré l'importance dans la manière dont la notion s'est disséminée - auxquels s'ajoute l'ouvrage de Bénédicte Shawlky-Milcent qui constitue assurément l'un des appuis les plus pertinents pour penser et mettre en œuvre la lecture subjective des élèves. À ces trois références, on peut adjoindre l'ouvrage fondateur d'A. Rouxel et G. Langlade et le livre de P. Bayard. On est en droit de s'interroger sur la présence de ce dernier car il ne relève pas de la didactique mais il a, lors de sa parution, reçu un accueil médiatique important, alimenté très vite des travaux et lectures stimulantes (Swoboda, Wierzbowska \& Wrońska, 2011); il fait partie des références grâce auxquelles et par lesquelles le concept de sujet lecteur s'est construit dans le champ de la didactique (Langlade \& Rouxel, 2008 ; Louichon, 2011, 2016 ; Dufays, 2013 ; Mazauric, Fourtanier \& Langlade, 2011a, 2011b) ; enfin, la séduction inhérente au texte bayardien est sans doute un argument pour le donner à lire ${ }^{20}$.

Cependant, l'enquête montre aussi que la notion a tendance à intégrer des éléments qui à priori n'en ressortissent pas (voir les sections II. 4 et II. 5), même si le questionnaire ne permet pas de juger pleinement de la manière dont les choses peuvent s'articuler dans le réel des pratiques de formation.

47 Force est de constater que le sujet lecteur en formation est devenu un concept "glouton » qui tend à subsumer une large part de ce qui relève de la didactique de la lecture littéraire, voire de la didactique de la littérature, alors qu'historiquement il en est le produit. Cette constatation s'inscrit dans la continuité de ce que nous avions observé relativement aux écrits des chercheuress en didactique : "Pour les auteurs, parler de lecture littéraire est une manière de parler d'un enseignement de la littérature dans lequel la place du lecteur est affirmée » (Louichon, 2011, p. 206).

\section{2 Du côté des pratiques}

48 Ainsi, si nous suivons les déclarations des formateurtrice's, nous pouvons constater que le concept de sujet lecteur construit dans le champ de la recherche, innerve bien et de manière massive les contenus et modalités de formation.

Il est évidemment tentant de chercher à voir ce qu'il en est du côté des pratiques effectives - et à fortiori ordinaires - d'enseignement/apprentissage de la littérature, ce que le format d'un article en revue ne nous permet pas. Néanmoins, nous proposons de mettre en lien ces conclusions avec quelques éléments tirés d'un projet de recherche portant sur les pratiques effectives de littérature en cycle 3 (Louichon, 2020). 
50 Les trois courts exemples que nous présentons sont constitués d'extraits d'entretiens d'auto-confrontation, à même de renseigner sur les préoccupations (Bucheton \& Soulé, 2009) des enseignant·e·s. Ces données permettent de faire émerger les représentations didactiques en jeu dans l'activité enseignante. Ce qui nous intéresse ici, c'est de mettre en lumière, avec toutes les réserves qui s'imposent, ce que nous pensons être des traces ou des échos de notre " concept glouton».

51 Un enseignant de $\mathrm{CM} 2^{21}$ travaille sur Rêves amers (Condé, 2001) et déclare :

La question que je me pose à l'heure actuelle, c'est que... C'est de savoir si je dois les amener au bout du livre, en fait. Voilà. Puisque... Euh... En fait, je ne peux pas maitriser ce que chaque enfant fera au niveau affectif de cette histoire-là qui... Qui se termine de façon dramatique, et qui est une réalité quotidienne. Euh... Du coup... Du coup, comme enseignant, je... je... je ne suis pas encore certain de, de les amener jusqu'au bout.

Donc tu ne leur laisserais pas lire la fin?

Je ne sais pas. Pas de... Je... Pour le moment, je n'ai pas la réponse. (c'est nous qui soulignons)

52 On constate ici que l'enseignant prend en compte l'élève comme sujet lecteur et cela l'amène à craindre l'impact psychoaffectif de la lecture et lui fait redouter l'absence de maitrise qui risque d'en résulter. Plus que tout se manifestent les hésitations clairement associées à un savoir-faire professionnel («comme enseignant») peu assuré.

Une enseignante de CM2 travaille sur Le Petit chaperon Uf (Grumberg, 2005). Elle déclare à propos d'une proposition d'élève manifestement erronée :

À ce moment-là il voit quelque chose qui, en tout cas moi je ne l'interprète pas du tout de cette manière-là. [...] Oui, non, là non tu as mal interprété. Je ne veux pas faire ça à ce moment-là donc j'essaie que ce soit le groupe.

Pourquoi tu ne veux pas faire ça?

Parce que ça reste de l'interprétation. On est au milieu du livre [...] Je ne veux pas venir à ce moment-là lui dire là non ce que tu as compris c'est pas ce qui, non je ne me vois pas faire ça. Peut-être que c'est tout le contraire qu'il faut faire, je ne sais pas. (c'est nous qui soulignons)

On voit bien ici que le sujet lecteur est pris en compte par l'enseignante mais la mécompréhension de l'élève se transforme en interprétation subjective acceptable. Le savoir didactique est peu assuré, comme en témoigne le discours même de l'enseignante.

55 Ces deux professeure's sont dans un inconfortable entredeux: il et elle sont conscientees de la dimension subjective de la lecture et cherchent même à la faire advenir. Dans le même temps, il et elle semblent démuniees quant à la manière de prendre en compte didactiquement la parole de l'élève lecteur.

Découvrons encore une parole d'enseignante de $6^{\mathrm{e}}$ :

Et après ce qui m'a intéressée dans la... ben dans le fait d'être filmée, de revenir, c'est que ça oblige à vraiment se questionner sur son rôle de prof et... justement sur la question de quand on a une interprétation de lecture, quelle place de liberté on laisse aux élèves et qu'est-ce qu'on impose. Et il y a toujours un équilibre à trouver entre pas imposer et en même temps faire passer des connaissances et des compétences, quoi [...]. Et donc, je trouve que c'est toujours... [elle rit]... c'est toujours... difficile.

57 Il ne s'agit pas ici de tirer des conclusions de quelques bribes recueillies auprès d'enseignante's mais plutôt de les considérer comme des échos de ce que nous avons 
développé. Les trois exemples de discours professoraux illustrent le fait que le sujet lecteur - même si le syntagme n'est pas utilisé comme tel - est bien devenu un concept avec lequel ils ou elles pensent leur enseignement. Indéniablement, du champ de la recherche à l'espace des recommandations, de la formation à l'enseignement, le concept se diffuse mais, ce faisant, il devient aussi plus diffus. Ce phénomène n'est évidemment en rien spécifique à ce concept-là car il semble inhérent à tout processus de dissémination conceptuelle ${ }^{22}$. Cependant, il nous apparait que cela peut parfois mettre les enseignante-s en situation difficile. Cela ne signifie pas que le concept de sujet lecteur doive être minoré en formation. Sans doute doit-il y être mieux circonscrit mais aussi configuré de sorte qu'il redonne aux enseignantees qui s'en saisissent la nécessaire maitrise professionnelle de gestion de la parole des élèves ${ }^{23}$.

\section{BIBLIOGRAPHIE}

AHR, S. (dir.) (2013). Vers un enseignement de la lecture littéraire au lycée. Expérimentations et réflexions. Grenoble : Scéren.

BARTHES, R. (1984) [1964]. Le Bruissement de la langue. Essais critiques IV. Paris : Éditions du Seuil. BELHADJIN, A. (2009). « Nouvelles approches de la lecture au lycée professionnel ». In : Butlen, M. \& Houdart-Mérot, V. (dirs). Interpréter et transmettre la littérature aujourd'hui. Cergy-Pontoise : Université de Cegy-Pontoise/Centre de recherche textes et francophonies, p. 105-121. BELLEMIN-NOËL, J. (2001). Plaisirs de Vampire. Paris : Presses universitaires de France.

BUCHETON, D. \& SOULÉ, Y. (2009). « Les gestes professionnels et le jeu des postures de l'enseignant dans la classe : un multi-agenda de préoccupations enchâssées ». Éducation \& didactique 3 (3), p. 29-48. En ligne : https://journals.openedition.org/educationdidactique/543.

BURGOS, M. (1992). « Lecteurs experts, lecteurs convers. De quelques lectures en lycées professionnels et ailleurs ». Pratiques 76, p. 55-76. En ligne : https://www.persee.fr/doc/ prati_0338-2389_1992_num_76_1_1678.

CONDÉ, M. (2001). Rêves amers. Paris : Bayard.

DAUNAY, B. (2007a). «État des recherches en didactique de la littérature ». Revue française de pédagogie 159, p. 139-189. En ligne : https://journals.openedition.org/rfp/1175.

DAUNAY, B. (2007b). « Le sujet lecteur : une question pour la didactique du français ». Le Français aujourd'hui 157, p. 43-51. En ligne : https://www.cairn.info/revue-le-francais-aujourd-hui-2007-2page-43.htm.

DAUNAY, B., REUTER, Y. \& SCHNEUWLY, B. (dirs) (2011). Les Concepts et les méthodes en didactique du français. Namur : Presses universitaires de Namur.

DUFAYS, J.-L. (2011). «Quel enseignement de la lecture et de la littérature à l'heure des “compétences”?». Pratiques 149-150, p. 227-248. En ligne : https://journals.openedition.org/ pratiques/1747. 
DUFAYS, J.-L. (2013). « Sujet lecteur et lecture littéraire : quelles modélisations pour quels enjeux ?». Recherches \& Travaux 83, p. 77-88. En ligne : https://journals.openedition.org/ recherchestravaux/666.

DUFAYS, J.-L (2016). « La lecture littéraire, histoire et avatars d'un modèle didactique ». Tréma. revue internationale en sciences de l'éducation et didactique 45, p. 9-17. En ligne : https:// journals.openedition.org/trema/3486.

DUFAYS, J.-L., GEMENNE, L. \& LEDUR, D. (1996). Pour une lecture littéraire. 1, Approches historique et théorique, propositions pour la classe de français. Louvain-la-Neuve : De Boeck/Duculot.

FISH, S. (2007) [1980]. Quand lire c'est faire. L'autorité des communautés interprétatives. Trad. de l'anglais (États-Unis) par É. Dobenesque. Paris : Éd. les Prairies ordinaires.

GRUMBERG, J.-C. (2005). Le petit Chaperon Uf. Arles : Actes Sud.

Jouve, V. (2011). « Du miroir au mirage ». In : Mazauric, C., Fourtanier, M.-J. \& Langlade, G. (dirs). Le Texte du lecteur. Bruxelles : P. Lang, p. 51-62.

LANGLADE, G. (2001). «Et le sujet lecteur dans tout ça? ». Enjeux. Revue de formation continuée et de didactique $d u$ français 51-52, p. 53-62.

LANGLADE, G. \& FOURTANIER M.-J. (2007). « La question du sujet lecteur en didactique de la lecture littéraire ». In : Falardeau, E. et al. (dirs). La Didactique du français. Les voies actuelles de la recherche. Québec : Presses de l'université Laval, p. 101-123.

LANGLADE, G \& ROUXEL, A. (2008). « Des références critiques, pour quoi faire ? ». Le Français aujourd'hui 160, p. 53-60. En ligne : https://www.cairn.info/revue-le-francais-aujourd-hui-2008-1page-53.htm.

LOUICHON, B. (2011). « La lecture littéraire est-elle un concept didactique? ». In : Daunay, B., Reuter, Y., Schneuwly, B. (dirs). Les Concepts et les méthodes en didactique du français. Namur : Presses universitaires de Namur, p. 195-216.

Louichon, B. (2016). « Dix ans de Sujet lecteur ». In : Petitjean, A. (dir.). Didactique du français et de la littérature. Metz : Université de Lorraine, p. 403-422.

LOUICHON, B. (2017). « La question des modèles dans les recherches sur le sujet » lecteur. In : Massol, J.-F. \& Rannou, N. (dirs). Le Sujet lecteur-scripteur de l'école à l'université. Grenoble : Université Grenoble Alpes Éditions, p. 47-57.

Louichon, B. (dir.) (2020). Un texte dans la classe. Pratiques d'enseignement de la littérature au cycle 3 en France. Bruxelles : P. Lang.

MAZAURIC, C., FOURTANIER, M.-J., LANGLADE, G. (dirs) (2011a). Le Texte du lecteur. Bruxelles : P. Lang.

MAZAURIC, C., FOURTANIER, M.-J., LANGLADE G. (dirs) (2011b). Textes de lecteurs en formation. Bruxelles : P. Lang.

Ministère de l'Éducation nationale, de la Jeunesse et des Sports (2015). « Programmes d'enseignement du cycle des apprentissages fondamentaux (cycle 2), du cycle de consolidation (cycle 3) et du cycle des approfondissements (cycle 4) ». Bulletin officiel spécial $n^{\circ} 11$ du 26 novembre 2015. En ligne : https://www.education.gouv.fr/bo/15/Special11/MENE1526483A.htm.

Ministère de l'Éducation nationale, de la Jeunesse et des Sports (2018). « Annexe 2. Cycle de consolidation (cycle 3) ». Bulletin officiel $n^{\circ} 30$ du 26 juillet 2018. En ligne : https:// www.education.gouv.fr/bo/18/Hebdo30/MENE1820169A.htm. 
Ministère de l'Éducation nationale, de la Jeunesse et des Sports (2019b). « Programme de l'enseignement de français de la classe de seconde générale et technologique et de la classe de première des voies générale et technologique ». Bulletin officiel spécial $n^{\circ} 1$ du 22 janvier 2019. En ligne : https://www.education.gouv.fr/bo/19/Special1/MENE1901575A.htm.

PENLOUP, M.-C., CHABANOIS, M. \& JOANNIDÈS R. (2011). « La "posture" : effet de mode ou concept pour la didactique du français? ». In : Daunay, B., Reuter, Y., Schneuwly, B. (dirs). Les Concepts et les méthodes en didactique du français. Namur : Presses universitaires de Namur, p. 151-174.

PETITJEAN, A. (2014). « 40 ans d'histoire de la "lecture littéraire" au secondaire à partir de la revue Pratiques ». Pratiques 161-162. En ligne : http://journals.openedition.org/pratiques/2155.

PICARD, M. (1986). La Lecture comme jeu. Paris : Édition de Minuit.

REUTER, Y. (2006). « À propos des usages de Goody en didactique. Éléments d'analyse et de discussion ». Pratiques 131-132, p. 131-154. En ligne : https://www.persee.fr/doc/

prati_0338-2389_2006_num_131_1_2124.

ROSIER, J.-M. (2014). « Sur le sujet lecteur ». Enjeux. Revue de formation continuée et de didactique du français 88, p. 89-99.

ROUXEL, A. \& LANGLADE G. (dirs) (2004). Le sujet lecteur. Lecture subjective et enseignement de la littérature. Rennes : Presses universitaires de Rennes.

SPRENGER-CHAROLLES, L. (1982). « Ils ne sauraient plus lire ! ». Pratiques 35, p. 3-5. En ligne : https:// www.persee.fr/doc/prati_0338-2389_1982_num_35_1_2438.

SWOBODA, T., WIERZBOWSKA, E. \& WROŃSKA, O. (dir.) (2011). « Autour des “livres que l'on n'a pas lus" ». Cahier Erta $n^{\circ} 2$. En ligne : https://czasopisma.bg.ug.edu.pl/index.php/CE/issue/view/83.

TAUVERON, C. (dir.) (2002). Lire la littérature à l'école. Paris : Hatier.

VIBERT, A. (2011). « Faire place au sujet lecteur en classe : quelles voies pour renouveler les approches de la lecture analytique au collège et au lycée ? ». Ressources pour le collège et le lycée. Paris : Ministère de l'Éducation nationale. En ligne : http://eduscol.education.fr/lettres/ im_pdflettres/intervention-anne-vibert-lecture-vf-20-11-13.pdf.

VUILLEMIN, J.-C. (2017). « Foucauld archéologue : généalogie d'un concept ». Implications philosophiques. Espace de recherche et de diffusion. Mis en ligne le 10 oct. 2017. http:// www.implications-philosophiques.org/implications-epistemologiques/foucault-archeologuegenealogie-dun-concept/.

\section{ANNEXES}

\section{Annexe 1}

Le corpus prend en compte 69 réponses complètes. 


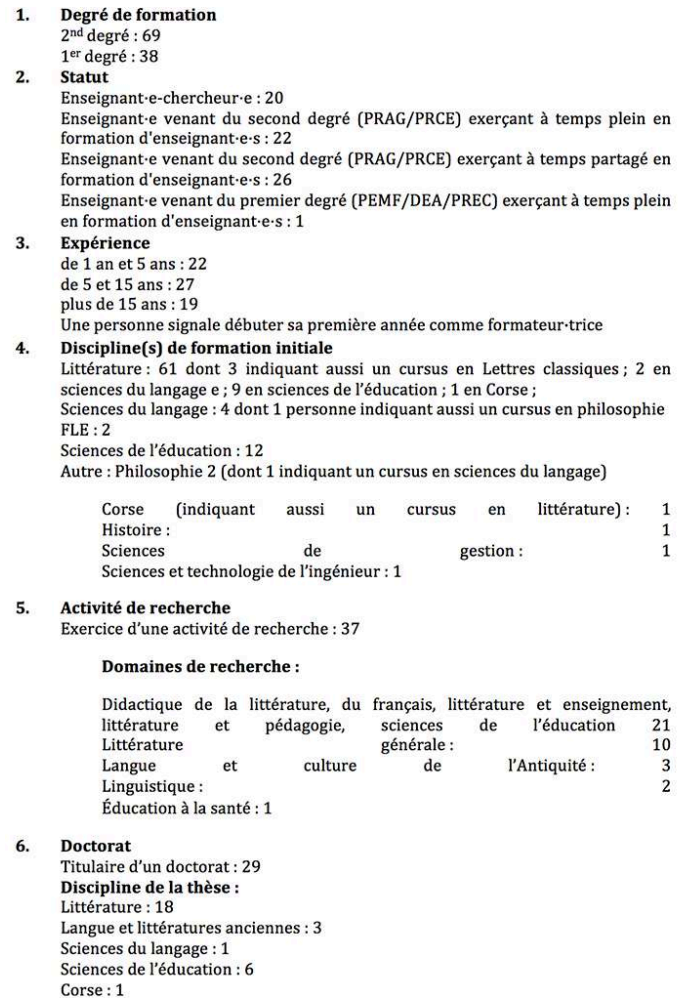

\section{Annexe 2}

\section{La bibliographie du questionnaire}

AHR, S. (dir.) (2018) [2013]. Vers un enseignement de la lecture littéraire au lycée. Expérimentations et réflexions. Grenoble : Scéren.

BAYARD, P. (2007). Comment parler des livres que l'on n'a pas lus?. Paris : Éditions de Minuit. BRUNEL, M., (2013). « Le sujet lecteur dans la classe : éléments pour un état des lieux des pratiques dans le secondaire ». Recherches \& Travaux 83, p. 117-130. En ligne : https:// journals.openedition.org/recherchestravaux/655.

CITTON, Y. (2007). Lire, interpréter, actualiser. Pourquoi les études littéraires ?. Paris : Amsterdam.

DAUNAY, B. (2007). « Le sujet lecteur : une question pour la didactique du français ». Le Français aujourd'hui 157 (2), p. 43-51. En ligne: https://www.cairn.info/revue-lefrancais-aujourd-hui-2007-2-page-43.htm.

DUFAYs, J.-L. (2006). «La lecture littéraire, des "pratiques du terrain" aux modèles théoriques ». Lidil. Revue de linguistique et de didactique des langues 33, p. 79-101. En ligne : https://journals.openedition.org/lidil/60.

DUFAYS, J.-L. (2017). « Analyser les pratiques d'enseignement-apprentissage de la lecture des textes littéraires : quelle modélisation pour quels enjeux ? ». Recherches en éducation 29, p. 11-21. En ligne: https://dial.uclouvain.be/pr/boreal/fr/object/ boreal\%3A187520/datastreams. 
DUMAYET, P. (2000). Autobiographie d'un lecteur. Paris : Pauvert.

ÉMERY-BRUNEAU, J. (2014). "La littérature au secondaire québécois: conceptions d'enseignants et pratiques déclarées en classe de français ». Lidil. Revue de linguistique et de didactique des langues 49, p. 71-91. En ligne: https://journals.openedition.org/lidil/ 3454 .

FALARDEAU, É. \& SAUVAIRE, M. (2015). « Les composantes de la compétence en lecture littéraire ». Le Français aujourd'hui 191, p.71-84. En ligne: https://www.cairn.info/ revue-le-francais-aujourd-hui-2015-4-page-71.htm.

LANGLADE, G. (2014). " La lecture subjective est-elle soluble dans l'enseignement de la littérature?». Études de Lettres 295, p.47-64. En ligne: http:// journals.openedition.org/edl/608.

LANGLADE, G. (2004). «Sortir du formalisme, accueillir les lecteurs réels ». Le Français aujourd'hui 145, p. 85-96. En ligne: https://www.cairn.info/revue-le-francais-aujourdhui-2004-2-page-85.htm.

LE GOFF, F. \& FOURTANIER, M.-J. (2017). Les formes plurielles des écritures de la réception. 2 vol. Namur : Presses Universitaires de Namur.

Louichon, B. (2016). « Dix ans de "Sujet Lecteur" ». In Petitjean A. (dir.). Didactiques du français et de la littérature. Metz : Université de Lorraine, p. 387-404.

MACÉ, M. (2011). Façons de lire, manières d'être. Paris : Gallimard.

MANGUEL, A. (2004). Journal d'un lecteur. Arles : Actes Sud.

MASSOL, J.-F., PlissonNEAU, G. \& BLOCH, B. (2017) (dirs). « Contextualiser et actualiser les œuvres littéraires au collège et au lycée ». Recherches \& Travaux 91. En ligne : http:// journals.openedition.org/recherchestravaux/922.

MAZAuric, C., Fourtanier, M.-J. \& LANGLAde, G. (dirs) (2011). Le Texte du lecteur. Bruxelles : P. Lang.

MERLIN-KAJMAN, H. (2016). Lire dans la gueule du loup. Essai sur une zone à défendre, la littérature. Paris : Presses universitaires de France.

ROUXel, A. \& LANGLAdE, G. (2004). Le Sujet lecteur. Lecture subjective et enseignement de la littérature. Rennes : Presses universitaires de Rennes.

ROUXEL, A. (2007). «Pratiques de lecture : quelles voies pour favoriser l'expression du sujet lecteur?». Le Français aujourd'hui 157, p. 65-73. En ligne : https://www.cairn.info/ revue-le-francais-aujourd-hui-2007-2-page-65.htm.

SHAWKY-MILCENT, B. (2016). La Lecture ça ne sert à rien. Usages de la littérature au lycée et partout ailleurs. Paris : Presses universitaires de France.

TODOROV, T. (2007). La Littérature en péril. Paris : Flammarion.

VIBERT, A. (2011). « Faire place au sujet lecteur en classe : quelles voies pour renouveler les approches de la lecture analytique au collège et au lycée? ". Ressources pour le collège et le lycée. Paris: Ministère de l'Éducation nationale. En ligne: http:// eduscol.education.fr/lettres/im_pdflettres/intervention-anne-vibert-lecturevf-20-11-13.pdf. 


\section{Annexe 3}

Voici une compilation des question posées. Le vrai questionnaire faisant 13 pages, nous avons choisi ici de ne pas faire figurer les propositions de réponses à choix multiple.

\section{Profil}

- Dans quel pays avez-vous exercé en 2017-2018?

- Dans quel(s) niveau(x) vos étudiantees ou stagiaires enseignent-ilelle·s ou enseigneront-ilelle's?

- Quel est votre statut professionnel

- Depuis combien de temps, formez-vous des maîtres?

- Quelle est votre formation?

-Exercez-vous des activités de recherche?

- Dans quelle(s) discipline(s)?

- Êtes-vous titulaire d'un doctorat?

- Dans quelle discipline?

\section{Formation et modalités de formation}

- La notion de sujet-lecteur est-elle présente dans vos formations?

- Quelle(s) modalité(s) de formation à la notion de sujet-lecteur utilisez-vous?

-Quelle(s) activité(s) d'enseignement-apprentissage en lien avec le sujet-lecteur proposez-vous?

- Quelle(s) référence(s) bibliographiques utilisez-vous pour construire votre formation sur le sujet-lecteur ?a

- Si vous le souhaitez-vous pouvez ajouter des références qui n'ont pas été mentionnées dans la bibliographie proposée.

- Quelle(s) référence(s) bibliographiques conseillez-vous à vos étudiants?

- Si vous le souhaitez, vous pouvez ajouter des références qui n'ont pas été mentionnées dans la bibliographie proposée.

- Avez-vous dirigé des mémoires en 2017-18?

- Combien de mémoires avez-vous dirigés en 2017-18?

- Dans combien de ces mémoires le sujet lecteur constitue-t-il un élément du cadre théorique?

\section{Commentaires personnels}

- Cet espace vous permet d'ajouter des informations qui vous semblent utiles.

\section{NOTES}

1. Article dont G. Langlade a souvent dit combien il avait compté dans son cheminement intellectuel.

2. Lequel, évidemment, s'inscrit aussi dans une filiation, des problématiques et des questionnements de recherche. L'importance des travaux de J.-L. Dufays (1996) et de 
Catherine Tauveron (2002), lesquels participaient d'ailleurs au colloque, doivent être rappelés.

3. On aura reconnu là les travaux menés respectivement par Nathalie Lacelle, Agnès Perrin-Doucey, Marion Sauvaire, Stéphanie Thieurmel, Bénédicte Shawlky-Milcent, Véronique Larrivé, Chloë Gabathuler, Pierre Moinard.

4. V. Jouve différencie de manière très utile le texte du lecteur comme compétence, à la suite d'U. Eco («l'encyclopédie du lecteur») ou de J.-L. Dufays («les systèmes de savoirs du lecteur »), et le texte du lecteur comme performance.

5. Pour mémoire, rappelons ce qu'écrivent R. Barthes (1970, p. 17) : «La subjectivité est une image pleine, dont on suppose que j'encombre le texte, mais dont la plénitude truquée n'est que le sillage de tous les codes qui me font, en sorte que ma subjectivité a finalement la généralité même des stéréotypes» ou S. Fish (2007, p. 74) : «Si l'ego est conçu, non comme une entité indépendante mais comme une construction sociale dont les opérations sont limitées par les systèmes d'intelligibilité qui l'informent, alors les significations qu'il confère au texte ne sont pas les siennes, mais trouvent leur source dans la (ou les) communauté interprétative(s) sur laquelle (ou lesquelles) il repose ».

6. Nous préfèrerons le terme de "notion " à celui de concept dès lors que l'on bascule du côté des usages car, comme le signalent B. Daunay, Y. Reuter et B. Schneuwly (2011, p. 16), « c'est plutôt leur usage [des concepts] dans certains travaux qui en diluent la valeur conceptuelle ».

7. Le cycle 2 est constitué des 3 premières années de l'école élémentaire, le cycle 3 comprend les deux dernières années de l'élémentaire et la première année de collège, le cycle 4 regroupe les trois années suivantes de collège. Ces trois cycles composent ce que l'on appelle parfois « l'école du socle ».

8. Pour un exposé plus substantiel, voir Louichon (2016).

9. À partir du logiciel LimeSurvey.

10. Enseignant·e-chercheure, enseignant $\cdot e$ du second degré à temps plein, comme à temps partagé.

11. Les chiffres/nombres entre parenthèses correspondent au nombre de participant-e's concernée's par l'information donnée.

12. Voir aussi l'information plus précise sur les profils des répondant·es exposée en annexe 1.

13. Appelé dorénavant Préparation.

14. Appelé dorénavant Étudiants.

15. L'item autre n'a jamais été choisi.

16. exp-hors recherche $=29$ membres ; exp-recherche $=21$ membres .

17. http://www.tropes.fr.

18. « lecture $»=21$; « lecteur $»=18$.

19. «lecture » $=14 ;$ « lecteur » $=7$.

20. Il est cependant évident que la posture critique adoptée par P. Bayard rend la compréhension de son œuvre parfois hasardeuse. Ainsi déclare-t-il « quelque chose est absolument déterminant dans ce que j'écris - le terme que je vais employer est très prétentieux, mais pour moi c'est un terme magnifique -, c'est l'humour. Je suis un 
écrivain qui se préoccupe de faire rire, ou au moins sourire, son lecteur " (Swoboda, Wierzbowska \& Wrońska, 2011, p. 258).

21. Dernière année de l'école primaire.

22. Par exemple, et pour rester dans le champ de la didactique du français, Penloup, Chabanois \& Joannidès (2011) sur le concept de " posture » ou Reuter (2006) à propos des concepts empruntés à J. Goody.

23. L'ouvrage issu du projet Talc (Louichon, 2020) développe cet aspect assez central dans la recherche menée.

a. Voir annexe 2

\section{RÉSUMÉS}

Depuis plus de 15 ans maintenant, le concept de sujet lecteur a été mis sur le devant de la scène en didactique de la littérature et a généré quantité de travaux de recherche. Cet article expose la manière dont il est devenu outillant, discuté, disséminé, vulgarisé et préconisé. Ce faisant, ce concept a ainsi migré du champ de la recherche à celui des recommandations. Nous cherchons précisément à appréhender la place qu'il prend depuis lors dans l'espace de formation en France. À cette fin, nous analysons des éléments de pratiques déclarées, recueillis à partir d'une enquête sous forme de questionnaires que nous avons réalisés auprès des formateurtrice's d'enseignante-s. Dans la conclusion, des données relatives à un projet de recherche portant sur les pratiques effectives d'enseignement de la littérature permettent de poser quelques questions relatives aux effets de cette dissémination du concept de sujet lecteur sur les pratiques enseignantes.

Since more than 15 years ago, the concept of the Sujet lecteur (reading subject) has been brought to the forefront in literature didactics, and has generated a great deal of research work. This article describes how it has became a tool discussed, disseminated, popularized and advocated. In doing so, the concept has thus moved from the field of research to that of recommendations. We seek precisely to understand the place it has since taken in the teacher training French space. To this end, we analyse elements of declared practices, collected from a survey in the form of questionnaires that we have carried out among teacher trainers. In the conclusion, data from a research project on actual literature teaching practices allow to ask a few questions about the effects of this dissemination of the concept of the Sujet lecteur on teaching practices.

\section{INDEX}

Mots-clés : Sujet lecteur, lecture littéraire, formation d'enseignant, didactique de la littérature

Keywords : Reading subject, literary reading, teacher training, didactics of literature 


\section{AUTEURS}

\section{BRIGITTE LOUICHON}

Université de Montpellier, Lirdef, F-34000 Montpellier, France

\section{AGNÈS PERRIN-DOUCEY}

Université de Montpellier, Lirdef, F-34000 Montpellier, France 